\title{
In Situ TEM for Rechargeable Batteries
}

\author{
Reza Shahbazian-Yassar ${ }^{1}$ \\ 1. Department of Mechanical and Industrial Engineering, University of Illinois at Chicago, Chicago, IL \\ 60607, United States.
}

The field of rechargeable batteries has been has received tremendous attention during the past 5-10 years due to vision of U.S. Department of Energy (DOE) in developing electrical cars and reduce environmental pollution. Traditional lithium-ion batteries are not able to fully meet the requirements set forth by DOE and other federal agencies in terms of energy density, power density, safety, and cost. Therefore, extensive research is undergoing in many universities and national labs to overcome these issues. An area of major efforts has been developing new materials to be used as electrode systems in order to boost the energy density or power density to reach the proposed goals. However, these efforts yet have not resulted in a breakthrough success in part due to complexity of electrochemical reactions happen in new materials and the lack of diagnostic tools to explain the link between performance, processing, and structure.

In situ Transmission Electron Microscopy has emerged as new imaging and analytical technique to monitor the electrochemical reactions happen inside rechargeable batteries. In this technique, one can set up a micron size electrochemical cell on the TEM sample holder where electrical signals can be applied to the cell using potentiostat or electrical source. Depending how the cell is constructed, one can either seal the electrochemical cell or leave it exposed to TEM vacuum column. The former situation is called "closed cell" and the later is known as "open cell".

In this talk, the PI will give an overview of his research on electrochemical batteries using open cell concept and some outlook for future design and research will be presented. Our team has looked into variety of materials including two-dimensionals (2Ds), one-dimensionals (1Ds), and zero-dimensionals (0Ds). A notable 2D material that we have investigated is phosphorene, which is a $2 \mathrm{D}$ form of phosphor layers isolated from bulk black phosphorous using chemical exfoliation. We studied the behavior of phosphorous against $\mathrm{Na}$ metal similar to a half-cell configuration. We were able to image the atomic structure of pristine phosphorous using an aberration-corrected scanning TEM. It was found that the pristine phosphorene has atomic stacking that enable anisotropic diffusion of $\mathrm{Na}$ ions across this $2 \mathrm{D}$ material. An interesting point was that the activation barrier energy for this transport was quite low and the sodiation happens at extremely fast speeds. The sodiation channels were imaged at atomic resolution using high angle annular dark field (HAADF) and electron energy loss spectroscopy (EELS) confirmed these sodiation channels.

Another system of interests has been alpha manganese dioxide $\left(\alpha-\mathrm{MnO}_{2}\right)$ nanowires. These materials can be used as both anodes or cathodes depending the voltage range that they are used or the mechanism by which they accommodate ionic exchange. The $\mathrm{MnO}_{2}$ nanowires were imaged in directions parallel and perpendicular to their zone axis and the atomic structure of the nanowires was reveled. Interestingly, this material posses two common tunnels known as $1 \times 1$ and $2 \times 2$, but the center of $2 \times 2$ channels are occupied by cations such as $\mathrm{K}$ atoms. We showed that surface energy plays a critical role during the synthesis of $\alpha-\mathrm{MnO}_{2}$ and (100) planes construct the outermost shell while some $2 \times 3$ or $2 \times 4$ defects along (110) may form.

The existence of the extra positive cation ions inside $2 \times 2$ tunnels of $\alpha-\mathrm{MnO}_{2}$ motivated us to study if lithium ions will be transported through $1 \times 1$ or through $2 \times 2$ tunnels. We observed that $2 \times 2$ tunnels are still the preferred tunnels but lithium insertion in $2 \times 2$ tunnels will result in two distinct crystal 
expansions that were detected by monitoring the dimensional changes of nanowires. These dimensional changes were associated with the insertion pathways of lithium ions around cation $\mathrm{K}$ ions existed in the tunnels. Such tunnels can accommodate sodium $(\mathrm{Na})$ ions too during the operation of room temperature $\mathrm{Na}$-ion batteries. Our study show that the diffusion of $\mathrm{Na}$ ions into the channels results in partial removal of $\mathrm{K}$ ions from the center of tunnels. We observed improvements in ionic transport and electrical conductivity of $\alpha-\mathrm{MnO}_{2}$ during the insertion of $\mathrm{Na}$ ions indicating a high cycling/rate capabilities in Naion batteries made of $\alpha-\mathrm{MnO}_{2}$ electrodes. These findings provide fundamental understanding for how alkaline ions transport within the solid state electrodes. 